\title{
The significance of chemokine CXCL-8 in esophageal carcinoma
}

Marta Łukaszewicz-Zając ${ }^{1}$, Sara Pączek ${ }^{1}$, Barbara Mroczko²

\author{
${ }^{1}$ Department of Biochemical Diagnostics, Medical University of Bialystok, Bialystok, \\ Poland \\ ${ }^{2}$ Department of Neurodegeneration Diagnostics, Medical University of Bialystok, \\ Bialystok, Poland
}

Submitted: 1 May 2017

Accepted: 22 August 2017

Arch Med Sci 2020; 16 (2): 475-480

DOI: https://doi.org/10.5114/aoms.2017.71933

Copyright $\odot 2020$ Termedia \& Banach

Chemokines are a group of small molecular weight proteins that are structurally related. These molecules play an important role in the growth, differentiation and activation of many types of cells $[1,2]$. Chemokines are synthesized mostly by leukocytes and act through their cognate G-protein coupled receptors to cause a cellular response, such as migration, adhesion or chemotaxis $[1,3]$. The chemokine family has been classified into four classes: CC, CXC, CX3C, and (X), based on the arrangement of $\mathrm{N}$-terminal cysteine residues [4]. These small peptides may also be grouped into inflammatory, homeostatic or dual function chemokines. Inflammatory chemokines can be induced during an immune response, whereas homeostatic chemokines are involved in control of cell migration [5]. The chemokine receptors are seven-transmembrane receptors coupled to G-proteins, that consist of an $\mathrm{N}$-terminus outside the cell surface, three extracellular and three intracellular loops as well as a C-terminus in the cytoplasm $[6,7]$.

C-X-C motif chemokine 8 (CXCL-8) is a pro-inflammatory cytokine that belongs to the CXC chemokine subgroup. The CXC family members, known as $\alpha$-chemokines, have an intervening amino acid between the first two cysteines and are located on chromosome 4 [8]. The CXC chemokines were further classified based on the presence or absence of the motif glu-leu-arg (ELR motif), into ELR+ and ELR- chemokines [3]. CXCL-8 is also known as interleukin-8 (IL-8) as well as neutrophil-activating peptide-1 (NAP-1). This cytokine was originally described as an inducer of neutrophil mobilizations in vivo $[9,10]$. The CXCL-8 gene encodes for a protein of 99 amino acids that is processed to either 72 amino acids in monocytes and macrophages or 77 amino acids in nonimmune cells [7, 11]. In physiological conditions CXCL-8 stimulates release of neutrophil granules. As a chemoattractant, this cytokine may change the levels of intracellular calcium as well as inducing re-arrangement of the cytoskeleton and exocytosis of granule proteins $[5,12]$. The activation of CXCL-8 is regulated via two cell-surface, $G$ protein coupled receptors (CXCR-1 and CXCR-2) that are structurally similar $[6,7]$. The CXCR-1 receptor is activated in response to binding of CXCL-8 and granulocyte chemotactic protein-2 (GCP-2), while CXCR-2 is activated by multiple C-X-C chemokines, such as growth-related oncogenes (GRO) [6, 7]. In addition, it has been assessed that CXCR-2 is the primary receptor for angiogenesis $[1,13]$.

Chemokines and their specific receptors are known to play an important role in inflammation, infection and tissue injury as well as in the

\author{
Corresponding author: \\ Marta Łukaszewicz-Zając \\ Department of \\ Biochemical Diagnostics \\ Medical University \\ 15 a Waszyngtona St \\ 15-269 Białystok, Poland \\ Phone: +48 857468785 \\ Fax: +48857468585 \\ E-mail: \\ marta.lukaszewicz-zajac@ \\ umb.edu.pl
}


development of cardiovascular and malignant diseases, including esophageal cancer [1, 3].

Esophageal cancer (EC) is one of the most aggressive tumors of the alimentary tract. Due to its unfavorable survival rate and late diagnosis, it is a focus of research for oncologists from all over the world [14]. The poor prognosis of EC patients is caused by the lack of early symptoms of disease [15]. At first, attention should be paid to signs of dysphagia, which starts with difficulty in swallowing. Other significant symptoms include vomiting, hemoptysis, chronic cough and epigastric pain [16]. Staging in EC is specified by the AJCC (American Joint Committee on Cancer) System, based on the TNM classification, which determines the primary tumor invasion ( $T$ factor), the participation of lymph nodes ( $\mathrm{N}$ factor) and distant metastases ( $M$ factor) [17]. There are two main histological subtypes of EC: esophageal squamous cell carcinoma (ESCC) and esophageal adenocarcinoma (EAC). As for the incidence, in 2012 EC occurred in an estimated 450000 cases worldwide, whereas ESCC occurred almost seven times more often than EAC. However, in Europe and the United States the number of EAC has been dramatically increasing for the last 20 years, while ESCC seems to be relatively stable in Western countries. It has been estimated that the rate of increase in EAC cases is greater than that of any other solid tumor over the same time period $[18,19]$. These changes in epidemiology might be due to the increasing frequency of Barrett's esophagus (BE) in the population. The factors which may affect ESCC are mostly observed in developing countries, while the components affecting EAC can be commonly observed in the economically developed countries $[1,18,19]$. It is generally known that the leading risk factors for both types of EC are tobacco smoking and excessive alcohol consumption as well as a bad economic situation and frequent intake of hot drinks. The most common factors which may cause EAC are obesity, gastroesophageal reflux disease (GERD) and BE [20]. In addition, some studies show that morbidity for men, especially for EAC cases, is higher than for women. Generally, it has been estimated that the mortality rate is similar to the incidence rate for EC [21]. The treatment guidelines include various options such as esophagostomy, radio- and chemotherapy or resection of local mucosa [22]. Despite the advances in EC management, the survival rate of EC patients is still poor, mostly because of the lack of effective screening for early detection and absence of early symptoms of the disease. Actually, there are no highly sensitive and highly specific biomarkers useful in early detection of EC [23]. The classical biochemical tumor markers used in the routine diagnosis and follow-up of EC patients are: for ESCC squamous cell carcinoma antigen (SCC-Ag), and for EAC carcinoembryonic antigen (CEA). However, their diagnostic sensitivity and specificity are still unsatisfactory [24]. Thus, currently there is an urgent need for novel, less invasive and cheaper screening tools for early EC diagnosis [25]. Currently, cancer proteomics provide a novel tool to identify new diagnostic biomarkers that may improve clinical outcome. In our previous studies we assessed the diagnostic usefulness of selected inflammatory proteins such as C-reactive protein (CRP), interleukin-6 (IL-6), chemokine CXCL12 and its specific receptor (CXCR-4) as well as the receptor for CXCL-8 (CXCR-2) in EC patients [26-29]. We conclude that the diagnostic significance of all analyzed inflammatory mediators was higher than that of classical tumor markers (CEA and SCC-Ag) of esophageal cancer and increased for combined measurement with CEA or SCC-Ag. In addition, serum CRP and IL- 6 levels were found to be indicators of tumor stage (TNM), the presence of lymph node ( $\mathrm{N}$ factor) and distant metastases ( $M$ factor) and may be useful in the assessment of tumor progression as well as prognosis of EC patients' survival. Our findings demonstrated the high diagnostic usefulness of the measurement of selected inflammatory mediators, especially in the diagnosis of patients with ESCC.

The role of chemokines and their receptors, including CXCL-8 and CXCR-2, in tumor development is explained by the fact that chronic inflammation is a crucial process in carcinogenesis. These proteins attract leucocytes to the inflammation site [3]. It has been shown that chemokines play a dual role in tumor progression. The recruitment of immune cells may promote anti-tumor activities via macrophages, which eliminate the tumor cells. On the other hand, the attraction of immune cells by chemokines and other factors secreted by malignant cells may facilitate the increase of tumor growth and supply survival factors as well as angiogenic mediators for the tumor. Thus, chemokines including CXCL-8 secreted by the tumor tissue or stroma promote leukocyte infiltration of the tumor $[3,30]$.

Originally, the chemokine CXCL-8 was identified as a neutrophil chemotactic factor in the supernatants of activated monocytes [31]. CXCL-8 as a CXC family chemokine attracts neutrophils and lymphocytes [3]. However, elevated expression of CXCL-8 and/or its receptors has been found in endothelial cells, infiltrating neutrophils, and tumor-associated macrophages as well as in cancer cells. Stromal cells are also able to produce CXCL8 to simplify the invasion or metastasis of cancer cells. Thus, it may suggest that this chemokine is a component of the tumor microenvironment $[32,33]$. Moreover, CXCL-8 may also contribute 
to human cancer progression due to its potential relevance as a mitogenic and angiogenic factor [31]. CXCL-8 was the first discovered angiogenic chemokine, so this cytokine is able to promote angiogenic responses in endothelial cells via stimulation of the proliferation, migration and survival of endothelial and cancer cells [7]. In addition, the levels of specific receptors for CXCL-8 are also up-regulated in malignant cells, which may cause the persistent presence of chemokines in the tumor microenvironment [3, 30].

The chemokine CXCL- 8 has been shown to play an important role in many steps of tumor progression, such as invasion, migration and proliferation of malignant cells [34-38]. Some clinical investigations have revealed the elevated expression and/ or blood levels of CXCL-8 in several human malignancies such as breast cancer $[39,40]$, colon cancer [41, 42], gastric cancer [43], ovarian cancer [44, 45], prostate cancer [46], pancreatic cancer [32], hepatocellular carcinoma [47] as well as esophageal carcinoma $[48,49]$. However, based on serum chemokine concentrations, little is known about the diagnostic significance of CXCL-8 as a potential tumor marker, especially for carcinoma of the esophagus.

The ability of the chemokine $\mathrm{CXCL}-8$ to regulate the communication between neoplastic cells and the tumor microenvironment facilitates tumor progression via growth and migration of neoplastic cells, regulation of angiogenesis and survival of tumor cells as well as the infiltration of leukocytes and modification of immune responses [48] (Figure 1). The levels of CXCL-8 correlated with platelet and neutrophil levels; thus these cells are sources of this cytokine, which may suggest a positive association between CXCL-8 and inflammation status in ESCC [49]. In addition, infiltrating neutrophils have also been shown to produce CXCL-8, so this cytokine may facilitate the progression of Barrett's esophagus and EAC [50]. However, the clinical relevance of CXCL-8 and its diagnostic significance as a tumor marker for EC are still poorly explained.
The diagnostic significance of CXCL-8 in esophageal cancer is presented in Table I. The study of Ogura et al. assessed the expression of CXCL-8 and its receptor (CXCR-2) in ESCC tissue as well as the association between immunoreactivity of this protein and clinicopathological characteristics of cancer using immunohistochemistry [48]. The authors indicated that elevated CXCL-8/CXCR-2 expression significantly correlated with the depth of invasion, lymph node metastasis as well as with lymphatic and venous invasion [48]. Moreover, the co-expression of CXCL-8 and its specific receptor (CXCR-2) was an independent predictive factor for recurrence-free survival of ESCC patients, which was assessed using Cox's hazards model [48]. The authors concluded that the overexpression of CXCL-8 and CXCR-2 may be useful as a potential diagnostic marker for selection of appropriate treatment as well as a predictor of outcome in ESCC patients [48]. Nguyen et al. assessed the expression of inflammation-related genes including CXCL-8 in EAC and non-tumor tissue using qRT$P C R$ (quantitative reverse transcriptase real-time PCR) [51]. The authors revealed that CXCL-8 gene expression was significantly increased in EAC and CXCL-8 expression levels in tumor and non-tumor samples were each associated with poor prognosis [51]. These findings suggest that elevated expression of inflammatory genes, such as CXCL-8, in association with miRNA levels, may predict clinical outcome for EC patients [51]. Thus, the authors confirmed the pro-tumoral role of this cytokine in EC.

The serum levels of CXCL-8 in EC patients have been scarcely investigated. It has been shown that the serum concentrations of CXCL-8 were significantly higher in ESCC patients in whom CXCL-8 and CXCR-2 were overexpressed [48]. The authors proved that $\mathrm{CXCL}-8$ secreted from ESCC cells is directly connected with elevated concentrations of serum CXCL-8 [48]. The study of Alvarez et al. demonstrated that serum levels of $\mathrm{CXCL}-8$ were significantly higher in EAC patients compared to
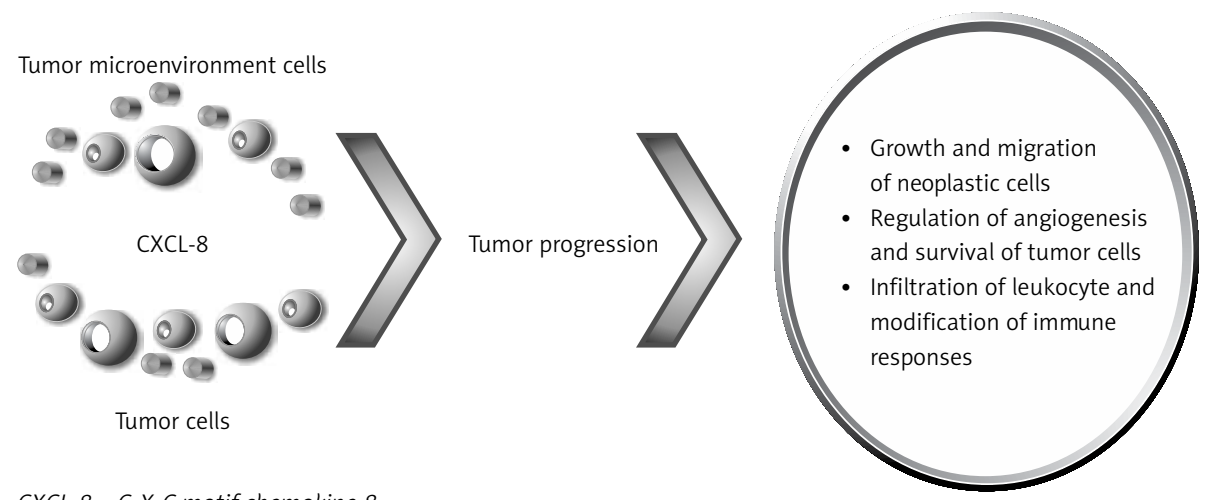

CXCL-8-C-X-C motif chemokine 8.

Figure 1. The role of chemokine CXCL-8 in cancer [48] 
Table I. Diagnostic significance of CXCL-8 in esophageal cancer

\begin{tabular}{|c|c|c|}
\hline Source & Results & References \\
\hline $\begin{array}{l}\text { Expression levels } \\
\text { (immunohistochemical } \\
\text { technique) }\end{array}$ & $\begin{array}{l}\text { - Elevated CXCL- } 8 \text { and its receptor (CXCR-2) expression significantly } \\
\text { correlated with the depth of invasion, lymph node metastasis and } \\
\text { with lymphatic and venous invasion } \\
\text { - Co-expression of CXCL- } 8 \text { and CXCR- } 2 \text { was an independent predictive } \\
\text { factor for recurrence-free survival of ESCC patients }\end{array}$ & [48] \\
\hline $\begin{array}{l}\text { Serum concentration } \\
\text { (ELISA method) }\end{array}$ & $\begin{array}{l}\text { - Serum concentrations of CXCL-8 were significantly higher in ESCC } \\
\text { patients in which CXCL-8 and CXCR-2 were overexpressed }\end{array}$ & \\
\hline $\begin{array}{l}\text { Expression levels } \\
\text { (qRT-PCR; microRNA) }\end{array}$ & $\begin{array}{l}\text { - CXCL-8 gene expression was significantly increased in EAC and CXCL-8 } \\
\text { expression levels in tumor and non-tumor samples were associated } \\
\text { with poor prognosis } \\
\text { - Elevated expression of CXCL-8 in association with miRNA could predict } \\
\text { clinical outcome for EAC patients }\end{array}$ & {$[51]$} \\
\hline $\begin{array}{l}\text { Serum concentration } \\
\text { (multiplex assay) }\end{array}$ & $\begin{array}{l}\text { - Serum levels of CXCL-8 were significantly elevated in EAC patients } \\
\text { compared to healthy controls }\end{array}$ & [52] \\
\hline $\begin{array}{l}\text { Serum concentration } \\
\text { (ELISA method) }\end{array}$ & $\begin{array}{l}\text { - Serum concentrations of CXCL-8 were significantly higher in ESCC } \\
\text { patients } \\
\text { - Serum CXCL-8 levels positively correlated with tumor size, cancer } \\
\text { dissemination, presence of lymph node and distant metastasis } \\
\text { - Serum CXCL-8 concentrations correlated with pro-inflammatory cells } \\
\text { and the biochemical marker of inflammation CRP }\end{array}$ & [49] \\
\hline
\end{tabular}

healthy controls, and this study was performed as a multiplexed assay on an ultra-sensitive chemiluminescence detection platform [52]. Similar findings have been presented by Krzystek-Korpacka et al., who demonstrated that serum concentrations of CXCL-8 were significantly higher in ESCC patients compared to healthy controls and correlated with tumor size and cancer dissemination [49]. In addition, serum CXCL-8 levels positively correlated with the presence of lymph node and distant metastasis [49]. However, there were no significant associations between serum $\mathrm{CXCL}-8$ concentrations and tumor stage of ESCC [49]. Moreover, serum CXCL-8 concentrations correlated with pro-inflammatory cells (platelets, leucocytes) and the biochemical marker of inflammation CRP. Thus, the authors proved that circulating CXCL-8 was associated with inflammatory status of ESCC. However, they concluded that detection rates were not satisfactory enough to allow for the recommendation of CXCL-8 measurement for clinical evaluation of ESCC patients [49]. In our previous study, we assessed the serum concentrations of the receptor for CXCL-8, CXCR-2, in EC patients in order to assess its clinical usefulness in the diagnosis and prognosis of EC patients compared to CRP, CEA and SCC-Ag. We indicated that serum concentrations of the receptor for CXCL-8 were significantly higher in EC patients compared to the control group, similarly to CEA and CRP levels. Moreover, concentrations of CXCR-2 were significantly higher in patients with poorly differentiated EC compared to well-differentiated tumors. The diagnostic sensitivity, accuracy and predictive value of negative results for serum
CXCR-2 concentrations were higher than for classical tumor markers (CEA and SCC-Ag), while being slightly lower than for CRP levels. The highest diagnostic sensitivity was found for the combined analysis of CXCR-2 and CRP.

The chemokine CXCL-8 is an activator of neutrophil chemotaxis and degranulation. Recent investigations have indicated that this chemokine plays a crucial role in development and metastasis of many tumors, including EC. In our paper, we review the current literature regarding the clinical significance of CXCL-8 in the diagnosis and prognosis of EC patients. The authors revealed the significantly increased expression of CXCL-8 in EC cells that correlated with clinicopathological tumor parameters. In addition, the measurement of CXCL-8 concentrations might also be useful in the diagnosis of this malignancy. Moreover, inhibition of its specific receptor, CXCR-2, may lead to decreased invasiveness of EC. In conclusion, current investigations have shed light on the potential usefulness of CXCL-8 in the diagnosis and prognosis of EC patients. However, due to its non-specific nature, future larger studies on serum of EC patients must be performed. Despite this, the CXCL8 and CXCL-8/CXCR-2 pathway may represent novel therapeutic applications.

\section{Acknowledgments}

The present project was supported by the Medical University of Bialystok, Poland (N/ST/ $M N / 16 / 001 / 2207)$. BM is supported by funds from the Leading National Research Centre (KNOW). 


\section{Conflict of interest}

The authors declare no conflict of interest.

\section{References}

1. Shrivastava MS, Hussain Z, Giricz O, et al. Targeting chemokine pathways in esophageal adenocarcinoma. Cell Cycle 2014; 13: 3320-7.

2. Borisch LC, Steinke JW. Cytokines and chemokines. J Allergy Clin Immunol 2003; 111 (2 Suppl): S460-75.

3. Raman D, Baugher PJ, Thu YM, Richmond A. Role of chemokines in tumor growth. Cancer Lett 2007; 256: 137-65.

4. Zlotnik A, Yoshie O. Chemokines: a new classification system and their role in immunity. 2000; 12: 121-7.

5. Palomino DC, Marti LC. Chemokines and immunity. Einstein 2015; 13: 469-73.

6. Holmes WE, Lee J, Kuang WJ, Rice GC, Wood WI. Structure and functional expression of a human interleukin-8 receptor. Science 1991; 253: 1278-80.

7. Waugh DJ, Wilson C. The interleukin-8 pathway in cancer. Clin Cancer Res 2008; 14: 6735-41.

8. Murphy PM, Baggiolini M, Charo IF, et al. International union of pharmacology. XXII. Nomenclature for chemokine receptors. Pharmacol Rev 2000; 52: 145-76.

9. Vandercappellen J, Van Damme J, Struyf S. The role of CXC chemokines and their receptors in cancer. Cancer Letters. 2008;267: 226-44.

10. Van Damme J, Van Beeumen J, Opdenakker G, Billiau S. A novel, $\mathrm{NH}$ 2-terminal sequence-characterized human monokine possessing neutrophil chemotactic, skin reactive, and granulocytosis-promoting activity. J Exp Med 1988; 167: 1364-76.

11. Brat DJ, Bellail AC, Van Meir EG. The role of interleukin- 8 and its receptors in gliomagenesis and tumoral angiogenesis. Neurooncology 2005; 7: 122-33

12. Paccaud JP, Schifferli JA, Baggiolini M. NAP-1/IL-8 induces up-regulation of CR1 receptors in human neutrophil leukocytes. Biochem Biophys Res Commun 1990; 166: 187-92.

13. Addison CL, Daniel TO, Burdick MD, et al. The CXC chemokine receptor 2, CXCR2 is the putative receptor for ELRC CXC chemokine-induced angiogenic activity. J Immunol 2000; 16: 5269-77.

14. Siegel R, Naishadham D, Jemal A. Cancer statistics, 2013. CA Cancer J Clin 2013; 63: 11-30.

15. Murakami S, Hashimoto T, Noguchi T, et al. The utility of endoscopic screening for patients with esophageal or head and neck cancer. Dis Esophagus 1999; 12: 186-90

16. Newton S, Hickey M, Brant J. Mosby's Oncology Nursing Advisor: A Comprehensive Guide to Clinical Practice. $2^{\text {nd }}$ ed. Mosby 2017; 58-60.

17. Jass JR, LH S. WHO International histological classification of tumors. Histological typing of intestinal tumors. Springer-Verlag, New York 1989.

18. Vial M, Grande L, Pera M. Epidemiology of adenocarcinoma of the esophagus, gastric cardia and upper gastric third. Recent Results Cancer Res 2010; 182: 1-17.

19. Bosetti C, Levi F, Ferlay J. Trends in oessophageal cancer incidence and mortality in Europe. Int J Cancer 2008; 122: 1118-29.

20. DomperArnal MJ, Ferrández Arenas Á, Lanas Arbeloa Á. Esophageal cancer: risk factors, screening and endoscopic treatment in Western and Eastern countries. World J Gastroenterol 2015; 14: 7933-43.
21. Arnold M, Soerjomataram I, Ferlay J, Forman D. Global incidence of oesophageal cancer by histological subtype in 2012. Gut 2015; 64: 381-7.

22. Berry MF. Esophageal cancer: staging system and guidelines for staging and treatment. J Thorac Dis 2014; 6 (Suppl 3): S289-97.

23. Merkow RP, Bilimoria KY, McCarter MD, Chow WB, Ko CY, Bentrem DJ. Use of multimodality neoadjuvant therapy for esophageal cancer in the united states: assessment of 987 hospitals. Ann Surg Oncol 2012; 19: 357-64.

24. Motoyama S, Miura M, Hinai Y, et al. CRP genetic polymorphism is associated with lymph node metastasis in thoracic esophageal squamous cell cancer. Ann Surg Oncol 2009; 16: 2479-85.

25. Uemura N, Kondo T. Current advances in esophageal cancer proteomics. Biochim Biophys Acta 2015; 1854 : 687-95.

26. Łukaszewicz-Zając M, Mroczko B, Kozłowski M, et al. Comparative evaluation of serum C-reactive protein (CRP) levels in the different histological subtypes of esophageal cancer (squamous cell carcinoma and adenocarcinoma of esophagus). J Clin Lab Anal 2012; 26: 73-81.

27. Łukaszewicz-Zając M, Mroczko B, Kozłowski M, et al. Higher importance of interleukin 6 than classic tumor markers (carcinoembryonic antigen and squamous cell cancer antigen) in the diagnosis of esophageal cancer patients. Dis Esophagus 2012; 25: 242-9.

28. Łukaszewicz-Zając M, Mroczko B, Kozłowski M, Szmitkowski $M$. The serum concentrations of chemokine CXCL12 and its specific receptor CXCR4 in patients with esophageal cancer. Dis Markers 2016; 2016: 7963895.

29. Łukaszewicz-Zając M, Kulczyńska-Przybik A, Muszyński P, Kozłowski M, Szmitkowski M, Mroczko B. Serum concentrations of receptor for interleukin 8 in patients with esophageal cancer. Pol Arch Med Wewn 2016; 126 : 854-61.

30. Balkwill F, Mantovani A. Inflammation and cancer: back to Virchow? Lancet 2001; 357: 539-45.

31. Xie K. Interleukin-8 and human cancer biology. Cytokine Growth Factor Rev 2001; 12: 375-91.

32. Chen Y, Shi M, Yu GZ, et al. Interleukin-8, a promising predictor for prognosis of pancreatic cancer. World J Gastroenterol 2012; 18: 1123-9.

33. Matsuo Y, Ochi $N$, Sawai $H$, et al. CXCL8/IL-8 and CXCL12/SDF-1alpha co-operatively promote invasiveness and angiogenesis in pancreatic cancer. Int J Cancer 2009; 124: 853-61.

34. Sethi G, Shanmugam MK, Ramachandran L, Kumar AP, Tergaonkar V. Multifaceted link between cancer and inflammation. Biosci Rep 2012; 32: 1-15.

35. Lazennec G, Richmond A. Chemokines and chemokine receptors: new insights into cancer-related inflammation. Trends Mol Med 2010; 16: 133-44.

36. Kruizinga RC, Bestebroer J, Berghuis P, et al. Role of chemokines and their receptors in cancer. Curr Pharm Des 2009; 15: 3396-416.

37. Iguchi H, Ono M, Matsushima K, Kuwano M. Overproduction of IL-8 results in suppression of bone metastasis by lung cancer cells in vivo. Int J Oncol 2000; 17 : 329-34.

38. Konno H, Ohta M, Baba M, Suzuki S, Nakamura S. The role of circulating IL-8 and VEGF protein in the progression of gastric cancer. Cancer Sci 2003; 94: 735-40.

39. Green AR, Green VL, White MC, Speirs V. Expression of cytokine messenger RNA in normal and neoplastic human breast tissue: identification of interleukin- 8 as 
a potential regulatory factor in breast tumors. Int J Cancer 1997; 72: 937-41.

40. König A, Vilsmaier T, Rack B, et al. Determination of interleukin-4, $-5,-6,-8$ and -13 in serum of patients with breast cancer before treatment and its correlation to circulating tumor cells. Anticancer Res 2016; 36: 3123-30.

41. Brew R, Erikson JS, West DC, Kinsella AR, Slavin J, Christmas SE. Interleukin-8 as an autocrine growth factor for human colon carcinoma cells in vitro. Cytokine 2000; 12: 78-85.

42. Xia W, Chen W, Zhang Z, et al. Prognostic value, clinicopathologic features and diagnostic accuracy of interleukin-8 in colorectal cancer: a meta-analysis. PLoS One 2015; 10: e0123484.

43. Kitadai Y, Haruma K, Sumii K, et al. Expression of interleukin-8 correlates with vascularity in human gastric carcinomas. Am J Pathol 1998; 152: 93-100.

44. Ivarsson K, Runesson E, Sundfeldt K, et al. The chemotactic cytokine interleukin-8 - a cyst fluid marker for malignant epithelial ovarian cancer? Gynecol Oncol 1998; 71: 420-3.

45. Yin J, Zeng F, Wu N, Kang K, Yang Z, Yang H. Interleukin-8 promotes human ovarian cancer cell migration by epithelial-mesenchymal transition induction in vitro. Clin Transl Oncol 2015; 17: 365-70.

46. Reiland J, Furcht LT, McCarthy JB. CXC-chemokines stimulate invasion and chemotaxis in prostate carcinoma cells through the CXCR2 receptor. Prostate 1999; 41: 78-88.

47. Chan SL, Chan AW, Chan AK, et al. Systematic evaluation of circulating inflammatory markers for hepatocellular carcinoma. Liver Int 2017; 37: 280-9.

48. Ogura M, Takeuchi $\mathrm{H}$, Kawakubo $\mathrm{H}$, et al. Clinical significance of cxcl-8/cxcr-2 network in esophageal squamous cell carcinoma. Surgery 2013; 154: 512-20.

49. Krzystek-Korpacka M, Matusiewicz M, Diakowska D, et al. Elevation of circulating interleukin- 8 is related to lymph node and distant metastases in esophageal squamous cell carcinomas: implication for clinical evaluation of cancer patient. Cytokine 2008; 41: 232-9.

50. Hannelien V, Karel G, Jo VD, Struyf S. The role of CXC chemokines in the transition of chronic inflammation to esophageal and gastric cancer. Biochem Biophys Acta 2012; 1825: 117-29.

51. Nguyen GH, Schetter AJ, Chou DB, et al. Inflammatory and microRNA gene expression as prognostic classifiers of Barrett's associated esophageal adenocarcinoma. Clin Cancer Res 2010; 16: 5824-34.

52. Alvarez H, Opalinska J, Zhou L, et al. Widespread hypomethylation occurs early and synergizes with gene amplification during esophageal carcinogenesis. PLoS Genet 2011; 7: e1001356. 